\title{
Tel Erani, Israel: Reporte de la campaña arqueológica de 2018 y sus antecedentes
}

\author{
lanir Milevski ${ }^{\mathrm{a}, \mathrm{b}}$, Marcelo Campagno ${ }^{\mathrm{c}, \mathrm{d}}$, Bernardo Gandullac, \\ Pablo Jarufc, d, M. Belén Daizoc,e, Marcin Czarnowicz, \\ Agnieszka Ochał-Czarnowiczf, Jacek Karmowskif, Dmitry \\ Yegorova , Eli Cohen-Sassong y Yuval Yekutielis
}

Fecha de recepción: 7 de julio de 2019. Fecha de aceptación: 1 de agosto de 2019.

\section{Resumen}

En el mes de julio de 2018, un equipo de investigadores del Instituto de Historia Antigua Oriental "Dr. Abraham Rosenvasser" en el marco del Proyecto PICTRaíces 2015-2943, participó en las excavaciones de la campaña arqueológica realizada en Tel Erani, Israel. Este sitio, excavado desde la década del ' 50 del siglo pasado, es clave para comprender las relaciones entre el sur de Palestina (Levante meridional) y Egipto durante la Edad del Bronce Antiguo IB (segunda mitad del $\mathrm{IV}^{\circ}$ milenio a.C.), pues se ha hallado una presencia significativa de cultura material egipcia, incluyendo un tiesto con un serekh del rey Narmer, así como también cerámica de un estilo local característico, llamada Erani C, que ha podido ser identificada en el delta del Nilo (Tell el-Farkha) y en tumbas protodinásticas, como la denominada U-j de Abidos. Parte de estos hallazgos se relaciona con la presencia de por lo menos dos murallas superpuestas que posiblemente rodeaban la totalidad del tel, de aproximadamente 25 ha, por lo que se trataría de uno de los asentamientos fortificados más tempranos de Palestina. En la campaña del año 2018 se excavaron dos áreas: el Área D3, donde abunda el material egipcio junto con elementos locales, y el Área P-Q, correspondiente a una de las zonas donde se encuentran las fortificaciones. Los 
resultados de esta última campaña indican que estas murallas serían anteriores a la fase egipcia, es decir el Bronce Antiguo IB1, pero luego, durante el Bronce Antiguo IB2, las relaciones entre ambas regiones se habrían intensificado, con la posibilidad de que los egipcios hayan pasado a tener un rol más activo en Tel Erani.

Tel Erani, Israel: Report of the 2018 Season and Its Background

Keywords

Tel Erani Early Bronze IB Palestine Egypt fortification walls

\section{Abstract}

In July 2018, a team of researchers from the Institute of Ancient Near Eastern History "Dr. Abraham Rosenvasser", in the framework of the Project PICTRaíces 2015-2943, participated in the excavations at Tel Erani, Israel. Tel Erani, excavated since the 1950's, is a key site to understand the relations between southern Palestine (Southern Levant) and Egypt during the Early Bronze IB (second half of the 4th millennium BC), since a significant presence of Egyptian findings has been found, including a sherd with a serekh of King Narmer. Furthermore, pottery of a characteristic local style, called Erani C has been found, identified also at the Nile Delta (Tell el-Farkha) and in protodynastic tombs, such as $\mathrm{U}-\mathrm{j}$ in Abydos. Parts of these finds are related to at least two overlapping fortification walls that possibly surrounded the entire tel which occupied approximately 25 ha. This would be one of the earliest fortified settlements in southern Palestine. In the campaign of 2018, two areas were excavated: Area D3, where Egyptian material is abundant along with local elements, and Area P-Q, corresponding to one of the areas where the fortifications are located. The results of this last campaign indicate that these defensive walls would be prior to the Egyptian phase, i.e. during the Early Bronze IB1. During the late phase, Early Bronze IB2, the relations between both regions would have intensified, with the possibility that the Egyptians have played a more active role at Tel Erani.

En el mes de julio de 2018, un equipo argentino, compuesto por investigadores del Instituto de Historia Antigua Oriental “Dr. Abraham Rosenvasser” (a partir de ahora IHAO), de la Facultad de Filosofía y Letras de la Universidad de Buenos Aires, formó parte de una nueva campaña arqueológica, en el sitio de Tel Erani, actual Estado de Israel. Tal participación se realizó en el marco del proyecto PICT-Raíces 2015-2943, titulado "Relaciones entre Egipto y Palestina a fines del IVo milenio a.C.: hacia la construcción de un modelo multidisciplinario de interpretación", dirigido por Marcelo Campagno, Ianir Milevski y Bernardo Gandulla. Dicho proyecto cuenta con la financiación de la Agencia Nacional para la Promoción Científica y Tecnológica (ANPCyT), dependiente de la ahora Secretaría de Ciencia, Tecnología e Innovación Productiva de la Nación Argentina.

El objetivo del citado proyecto es determinar con mayor precisión las características y los cambios en las relaciones entre el Egipto Protodinástico y Palestina 
a fines del $\mathrm{IV}^{\circ}$ milenio a.C. Entre las actividades programadas, destaca con particular relevancia el trabajo de campo en un sitio clave para dilucidar la naturaleza de estas relaciones, condición que Tel Erani cumple con creces, no sólo por el hallazgo de amplias evidencias sobre presencia egipcia, sino también por el hecho de que cerámicas producidas en esta región fueron identificadas en tumbas del Alto Egipto (Hartung, 2002; Dreyer, 2011) y sitios del delta del Nilo (Czarnowicz, 2018).

Las excavaciones en este sitio están dirigidas en la actualidad por tres entidades: la Universidad Jaguelónica de Cracovia, ${ }^{1}$ la Universidad Ben-Gurión del Néguev (Beersheba), y la Autoridad de Antigüedades de Israel (IAA), con la colaboración de la Universidad de Buenos Aires. El equipo argentino se completó con Pablo Jaruf y M. Belén Daizo, y contó con la colaboración de Alejandro Mizzoni, y de los estudiantes de grado Ariel Castro Nessim y Ezequiel Cismondi. Dicha participación se dividió en dos áreas: Área D3, correspondiente a la fase con presencia egipcia; y el Área P-Q, la cual constituye una ampliación de un área abierta en la temporada previa (2015-2016). Esta última consistió en un trabajo de rescate con motivo del ensanchamiento de una ruta que pasa exactamente al sur del sitio, y estuvo codirigida por Dmitry Yegorov y por Ianir Milevski.

\section{Antecedentes I: de las primeras excavaciones hasta 2015}

El sitio de Tel Erani (Tell esh Sheik Ahmed el 'Areini) se encuentra en el borde oriental de la llanura costera mediterránea, en el límite con la zona de piedemonte conocida como Shefelah (Fig. 1). El tel es bien visible en el paisaje de la planicie y ha sido investigado desde fines de los ' 50 por Samuel Yeivin, entonces director del Departamento de Antigüedades de Israel (Fig. 2). En esa época se creía que el sitio era la ciudad filistea de Gat, pero luego de varias campañas se descartó esta posibilidad, pues era muy poca la cerámica hallada capaz de ser atribuida a los filisteos (Yeivin, 1975). ${ }^{2}$

En estas primeras excavaciones se determinaron dos cuestiones centrales respecto de los estratos que se atribuyeron al Bronce Antiguo I (a partir de ahora BA), es decir, hacia la segunda mitad del IV ${ }^{\circ}$ milenio a.C.: 1) la existencia de lo que parecía ser una muralla de ladrillos en el Área N, al NE del sitio; 2) la presencia de cerámica egipcia correspondiente a la fase Nagada II y III en el Área $\mathrm{D}$, al Sudoeste del sitio, además de un serekh, en el que se ha reconocido el nombre de Narmer, primer rey de la Dinastía I en Egipto ${ }^{3}$ (Fig. 3). A partir de entonces, se ha debatido sobre la posibilidad de postular la existencia de una colonia egipcia con centro en la zona de Tel Erani o en Erani mismo, o por lo menos una intensificación de las relaciones entre el valle del Nilo y el sur del Levante (Brandl, 1989; Anđelković, 1995; Debowska-Ludwin et al., 2012; Greenberg, 2014).
1. La participación de los investigadores de la Universidad Jaguelónica de Cracovia se realizó en el marco del proyecto financiado por el Centro Nacional de Ciencia de Polonia (UMO-2016/23B/HS3/01886).
2. En la actualidad existe un debate sobre la llamada cerámica filistea (e.g. Dothan y Zuckermann, 2015) y su adscripción al pueblo filisteo tal cual aparece en la Biblia. Sobre este hemos realizado una reciente exposición (Milevski et al., 2018) relativa al cementerio de la Edad del Hierro en el sitio (ver más abajo al respecto).

3. Este serekh fue hallado en el estrato $V$ según Yeivin, pero Braun (2009) pone en discusión esta datación, sosteniendo que no existen precisiones en los informes originales de la excavación, por lo que recomienda considerarlo simplemente como post-Erani C (véase la nota 3). 


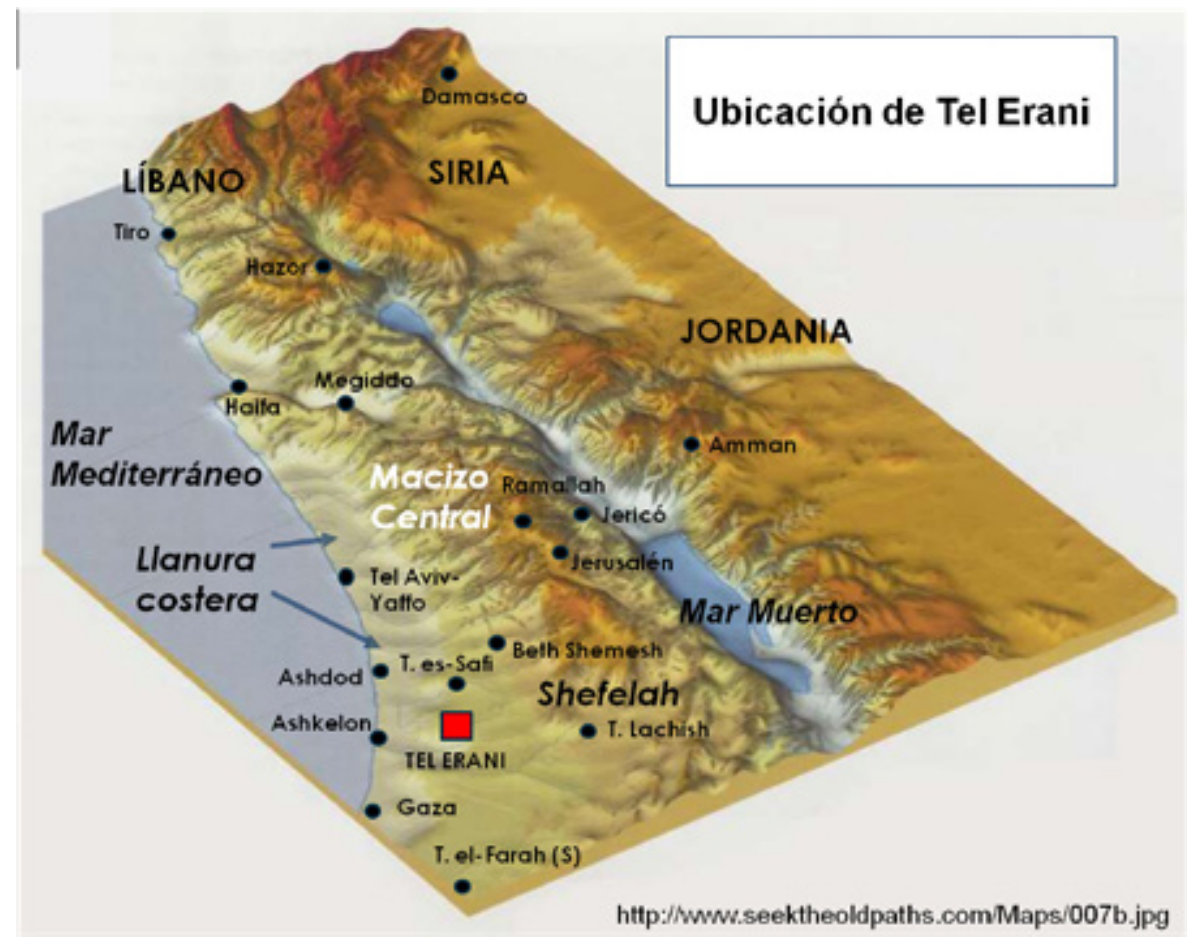

Fig. 1. Ubicación del sitio arqueológico Tel Erani, Israel

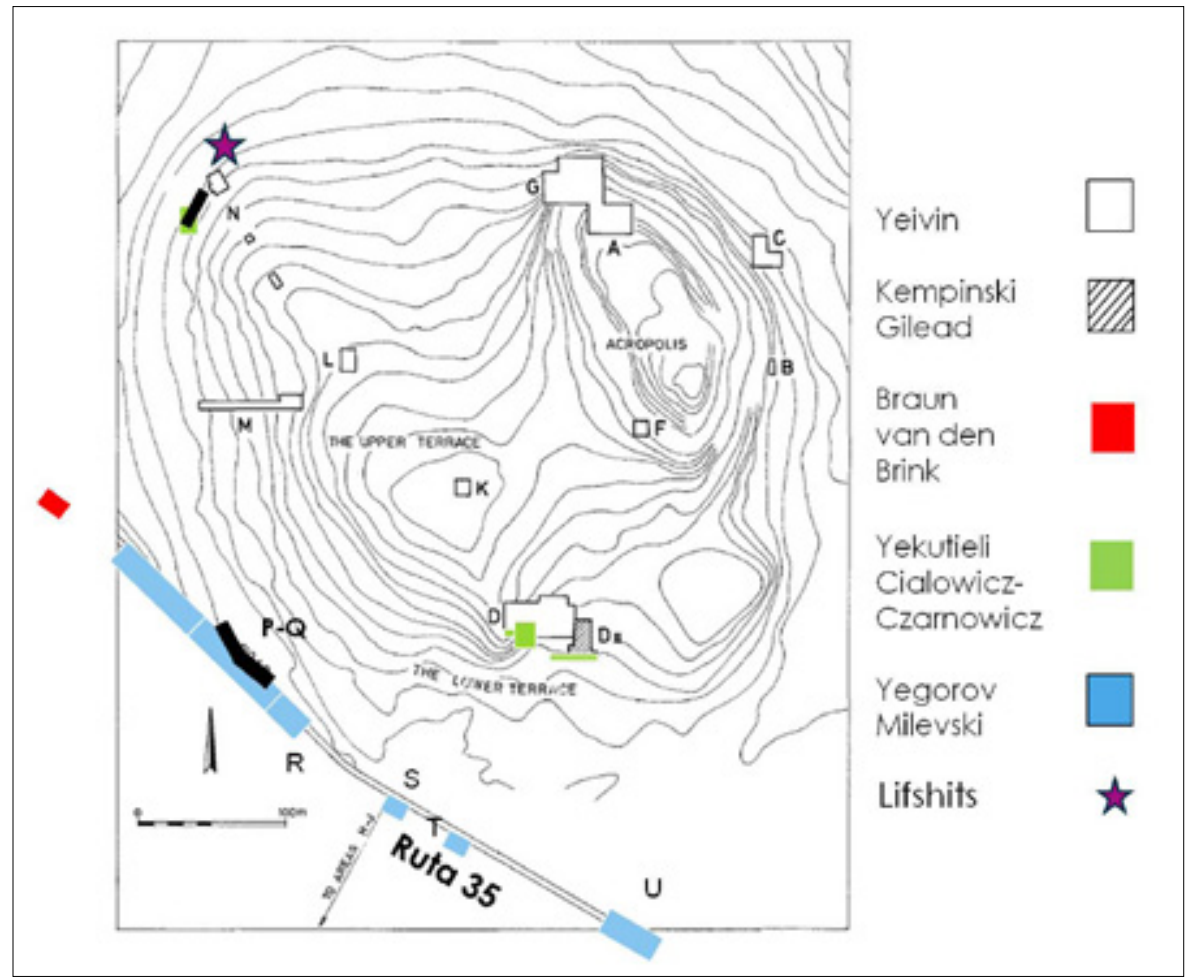

Fig. 2. Plano de las áreas excavadas de Tel Erani (tomado de Milevski et al., 2016) 
Yeivin también excavó una estructura con pilares (edificio $n^{\circ} 7132$ ), de aparente función pública, categorizada más tarde como "proto-palacio" (de Miroschedji, 2012-2013). Posteriormente, en los años ' 80 , una nueva misión de la Universidad de Tel Aviv y de la Universidad Ben-Gurión del Néguev dirigida por Aharon Kempinski e Isaac Gilead, excavó una estructura similar en el Ârea DII (edificio $\mathrm{n}^{\circ} 232$ ) (Fig. 4). La cerámica relacionada con esta última, característica del sitio, y denominada a partir de entonces como Erani $C,{ }^{4}$ indicaría que es anterior a la presencia egipcia, ubicándose entre el 3400 y el 3200 a.C. aproximadamente (Yekutieli, 2006). A partir de entonces, la segunda parte del BA en el sur de Palestina se subdivide en dos fases: BA IB1 u Horizonte Erani C, y BA IB2, correspondiente a la presencia egipcia (Tabla 1).

\begin{tabular}{|c|c|c|}
\hline Años a.C. & Palestina & Egipto \\
\hline $3300-3100 / 3000$ & BA IB2 & Nagada IIIB-C \\
\hline $3500 / 3400-3300 / 3200$ & BA IB1 & Nagada IID-IIIA \\
\hline $3700 ?-3500 / 3400$ & BA IA & Nagada IIB-C \\
\hline
\end{tabular}

Tabla 1: Cronología comparada de Tel Erani con los períodos de Palestina y Egipto (adaptado de Yekutieli, 2000, 2006; Czarnowicz, 2018).

En la segunda década de los años 2000, la Universidad Jaguelónica de Cracovia y la Universidad Ben-Gurión del Néguev iniciaron un nuevo proyecto bajo la dirección de Yuval Yekutieli, Marcin Czarnowicz y Krystof Ciałowicz, el cual tuvo como objetivo explorar la relación entre las distintas fases, sincronizar los estratos con cerámica egipcia y local, y establecer la naturaleza de las relaciones con Egipto. Las excavaciones se centraron en el Área N y en el Área D. En el Área N continuaron excavando partes de la muralla ${ }^{5}$ de ladrillos identificada en tiempos de Yeivin, llegando a la conclusión de que debía ser datada en el Horizonte Erani C, es decir, previa a la fase egipcia. Por otra parte, el Área D se reabrió en 2013 siendo uno de los principales objetivos reconsiderar las afirmaciones de Yeivin sobre la presencia egipcia en el sitio (Czarnowicz et al., 2016). Las nuevas trincheras fueron llamadas Área D3, para diferenciarlas de las dos épocas anteriores en las que ya se había trabajado sobre la ladera sur de Tel Erani. El espacio de trabajo fue dividido en dos: $\mathrm{D} 3 \mathrm{H}$ (en la parte superior) y D3L (en la parte inferior).
4. La denominación de Erani C deriva de los niveles determinados por Kempinski y Gilead (1991) en sus excavaciones de la década del '8o. El nivel A correspondía a estratos desde el período Bizantino hasta el Bronce Tardío; el B a la Edad del BA II-III; el C al gran edificio $n-232$; y el $D$ a un estrato de la primera parte del BA I.

5. Cabe aclarar que empleamos aquí indistintamente murallas y fortificaciones. Si bien el término fortificación puede incluir, además de murallas, otro tipo de elementos defensivos, en el caso de las construidas en ladrillo no siempre resulta posible delimitar de manera exacta las partes que la componen. 


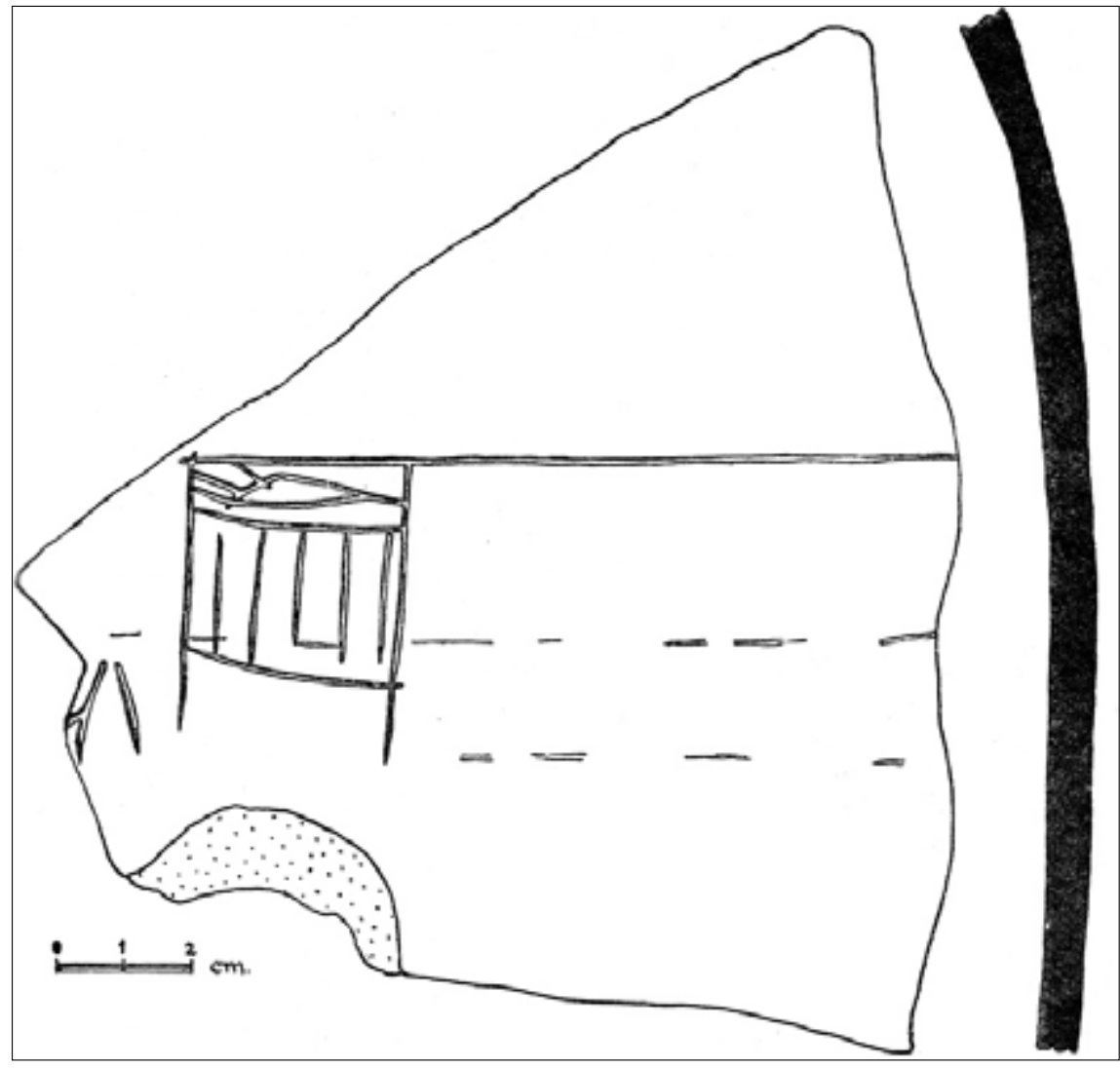

Fig. 3. Serekh de Narmer hallado en Tel Erani (tomado de Yeivin, 1960)

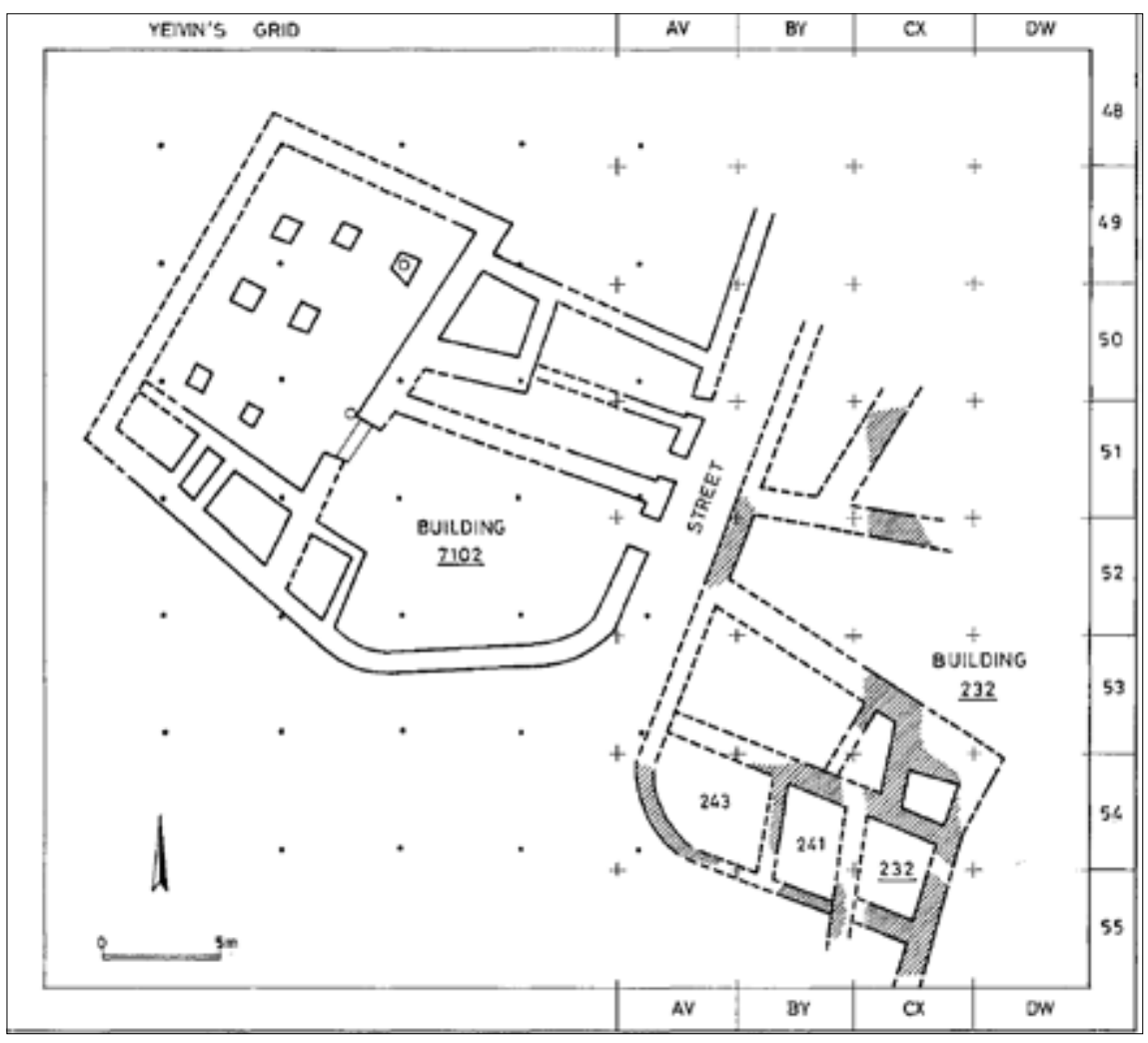

Fig. 4. Edificios no 7102 y $n^{\circ} 232$ excavados en el Área DII (tomado de Kempinski y Gilead, 1991) 
Durante la primera temporada de trabajo en la cuadrícula J12 ubicada en D3H se hallaron los restos de una pared, un horno ubicado detrás, dos pozos y un piso. En el contexto de estas estructuras se encontró cerámica Nagada, dentro del horno un cuchillo de pedernal egipcio y varias piedras planas que rellenaban uno de los pozos. Dichos rasgos son conocidos tanto en Egipto, asociados con la cultura Nagada, como en sitios del Levante meridional relacionados con presencia egipcia (e.g. Gophna, 1995), donde parecen haber sido utilizados para secar moldes de pan (bread-baking moulds). Estos hallazgos fueron interpretados como un claro indicio de grupos nilóticos en Tel Erani, por lo tanto, se tomó la decisión de ampliar esta área de trabajo en las siguientes excavaciones.

En las temporadas 2014-2015 se halló gran parte de un complejo consistente en un edificio casi cuadrado designado como $\mathrm{H}-4$, que habría sido utilizado con propósitos de almacenamiento y preparación de alimentos. Los hallazgos en dicha área que fueron datados en el BA IB2 se asignaron a la fase 6 de Tel Erani, que podría ser la última etapa con presencia egipcia en el sitio. También pudo delimitarse una estructura anterior a la construcción de este edificio, quizás vinculada con presencia nilótica, asignada a la fase 8 , pero sólo fue excavada de manera parcial. Entre ambos edificios se distingue una fase intermedia (7) con restos arquitectónicos muy mal conservados y numerosos pozos, algunos de los cuales se utilizaron como hornos de pan, como indica el hallazgo de cerámica in situ.

\section{Antecedentes II: La campaña de 2015-2016}

Como dijimos al comienzo, producto de la ampliación de la ruta que corre al sur del tel, la Autoridad de Antigüedades de Israel emprendió una campaña de rescate desde el mes de octubre de 2015 hasta febrero de 2016 (Milevski et al., 2016). Cabe señalar que, si bien en principio se abrieron dos Áreas, P y Q, al final ambas se juntaron, por lo cual se decidió identificarlas como una sola: Área P-Q.

Durante esta campaña se excavaron alrededor de $2500 \mathrm{~m} 2$ (unas 50 cuadrículas de $5 \times 5 \mathrm{~m}$ cada una), donde se identificaron varios estratos datados del BA IB, y un cementerio de la Edad de Hierro IB-IIA, además de material tardío hasta la época otomana.

Lo más significativo de los hallazgos correspondientes al BA IB es que, en su mayoría, representan de manera clara el Horizonte Erani C, BA IB1, con excepción de algunos pozos que contienen material del BA IB2, es decir, del período de la presencia egipcia en el sitio. Tres estratos en la parte inferior de los niveles excavados presentan recintos habitacionales construidos en ladrillos de barro con cerámica, instrumentos de pedernal, huesos de animales y conchillas de madreperla. En varios casos se encontraron pisos repletos de material cerámico y orgánico que parecen haber sufrido un episodio de incendio y parcial destrucción (Milevski et al., 2016: 47-49).

Sobre estos restos se construyeron dos niveles de murallas que fueron numeradas, de la inferior a la superior, como W200 y W204. De lo que se conservó, 
se puede afirmar que la muralla inferior -W200- tiene al menos $5 \mathrm{~m}$ de ancho y que su altura no se conserva más de 1,5 m. La muralla superior -W204muestra un ancho de 2,5 m, conservando también una altura de 1,5 $\mathrm{m}$. Los sedimentos excavados contenían cerámica del Horizonte Erani C, por lo que se trataría, entonces, de la parte sur de la misma muralla excavada años antes en el Área N (ver Fig. 2).

En el extremo Oeste del área P-Q se tomaron muestras de semillas carbonizadas en tres de los estratos: dos inferiores, dentro de habitaciones (P 8-7), y uno superior que representa el sedimento acumulado por fuera de la muralla inferior -W200- (P 6). Las fechas obtenidas por radiocarbono, realizadas por Joanna Regev y Elisabetta Boaretto en los laboratorios del Instituto Weizmann de Ciencia (Israel), dieron el siguiente rango de inferior a superior (Fig. 5):

» Estrato P8: alrededor del 3250 cal. a.C.

» Estrato P7: alrededor del 3200 cal. a.C.

» Estrato P6: alrededor del 3200-3150 cal. a.C.

En dos pozos de la misma parte del área $\mathrm{P}-\mathrm{Q}$ apareció material local datado del BA IB2, más algunos tiestos de cerámica egipcia correspondientes a Nagada IIIB-C. Estos pozos contienen la misma cerámica que fue hallada en el Área D3, siendo la datación de esta última de alrededor del 3100 a.C. (ver más abajo), según las cronologías más recientes (Czarnowicz, 2016).

Es necesario aclarar que debido a que esta campaña correspondió a un trabajo de rescate, la misma contaba con límites fijados de antemano, por lo que no se podía exceder la zona predeterminada para la excavación. La campaña de 2018, ya con la presencia del equipo de investigadores del IHAO, se propuso entonces expandir esta área para trabajar en la parte interior de las murallas.

\section{La campaña de 2018: Área D3H}

El trabajo arqueológico en el Área D3H estuvo dirigido por Marcin Czarnowicz y Agnieszka Ochał-Czarnowicz, y contó con la supervisión de M. Belén Daizo (IHAO) (Fig. 6). Las tareas realizadas fueron divididas en dos instancias de registro y excavación: por un lado, en las banquetas (balks) (4 m x $1 \mathrm{~m})$, que fueron resguardadas como testigos de la estratigrafía de la cuadrícula, y por otro, en las respectivas cuadrículas ( $5 \mathrm{~m}$ x $5 \mathrm{~m}$ ).

Los objetivos para la temporada 2018 en esta área fueron finalizar la operación de remoción de banquetas y continuar los trabajos para exponer los restos de las fases 7 y 8 . 


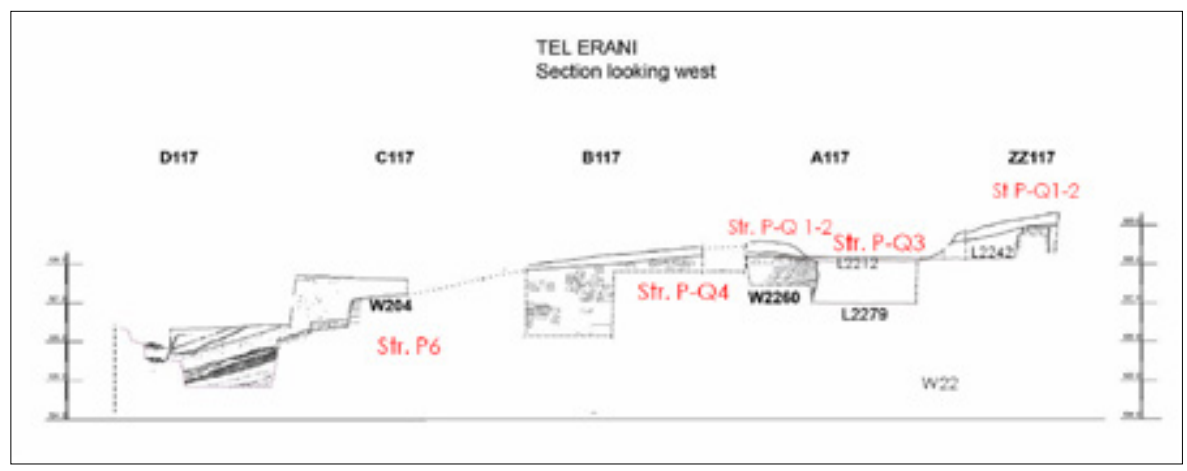

Fig. 5. Perfil occidental de la muralla excavada en el Área P-Q

(dibujo de M. Czarnowicz, Universidad Jaguelónica de Cracovia y cortesía del IAA)

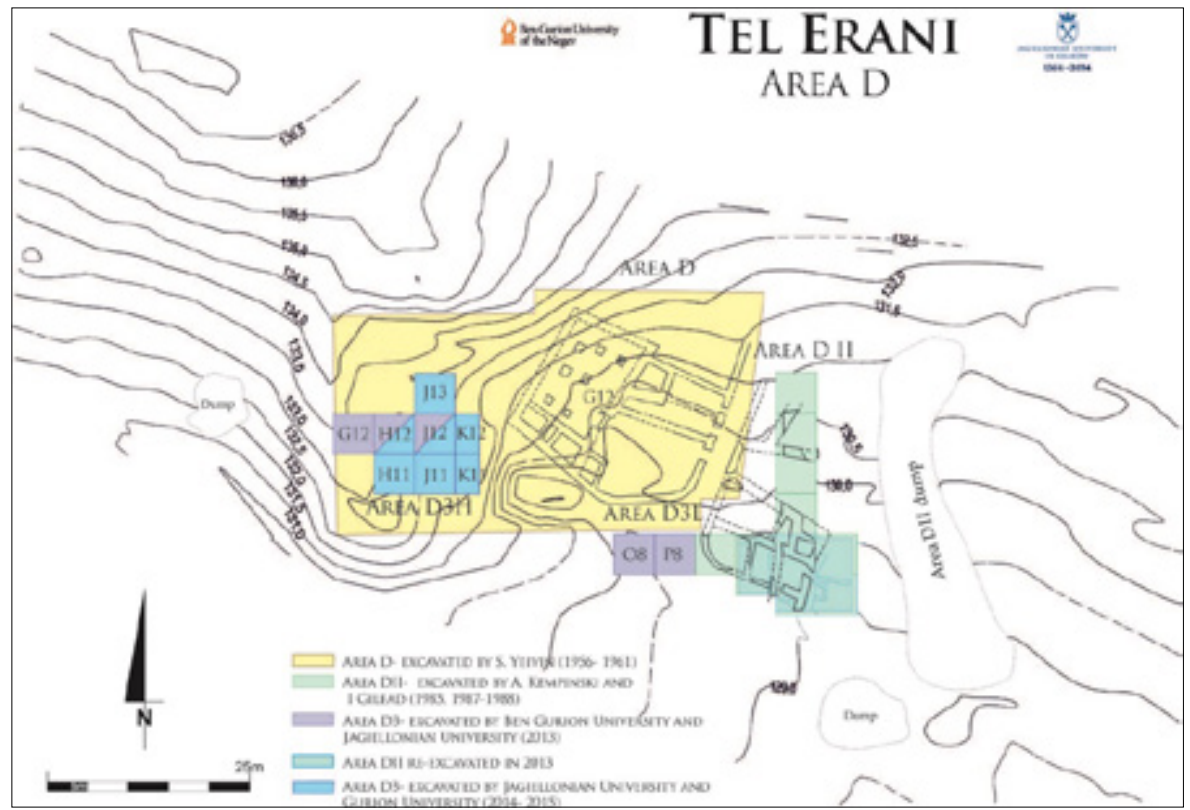

Fig. 6. Área D: cuadrículas trabajadas en las últimas campañas (cortesía de la Universidad de Ben-Gurión, Universidad Jaguelónica de Cracovia y del IAA)

Dentro de las banquetas, se identificaron los restos de las paredes y los pisos del edificio H-4. En la intersección de cuatro banquetas se conservó toda la sala lindante a la pared exterior del edificio H-4. Es posible interpretar la presencia de una escalera que conduce al piso superior del edificio. Dentro de la habitación se encontró una pequeña esfera de arcilla, posiblemente una ficha o token ${ }^{6}$ (Fig. 7) y la mayor parte del cuerno de un animal, probablemente un bóvido. En los pisos conectados al edificio $\mathrm{H}-4$ se encontraron muchos fragmentos de cerámica Nagada. En la parte interior, la mayoría de ellos pertenecían a diferentes tipos de cuencos, predominantemente pulidos a medias y "cuencos de loto" (lotus bowl). En la parte exterior, el conjunto difiere y consiste principalmente en recipientes de almacenamiento y moldes de pan. En ambos casos también se observó una cantidad significativa de cerámica local del BA IB2. Mientras que en varias partes de esta área la cerámica Nagada es dominante, en otras partes predomina la local.
6. Sobre este tipo de hallazgos cf. Kolodziejczyk, 2012; Czarnowicz et al., 2016: 30-31 (Área D3), y Milevski et al., 2016: 55-56 (Área P-Q). 

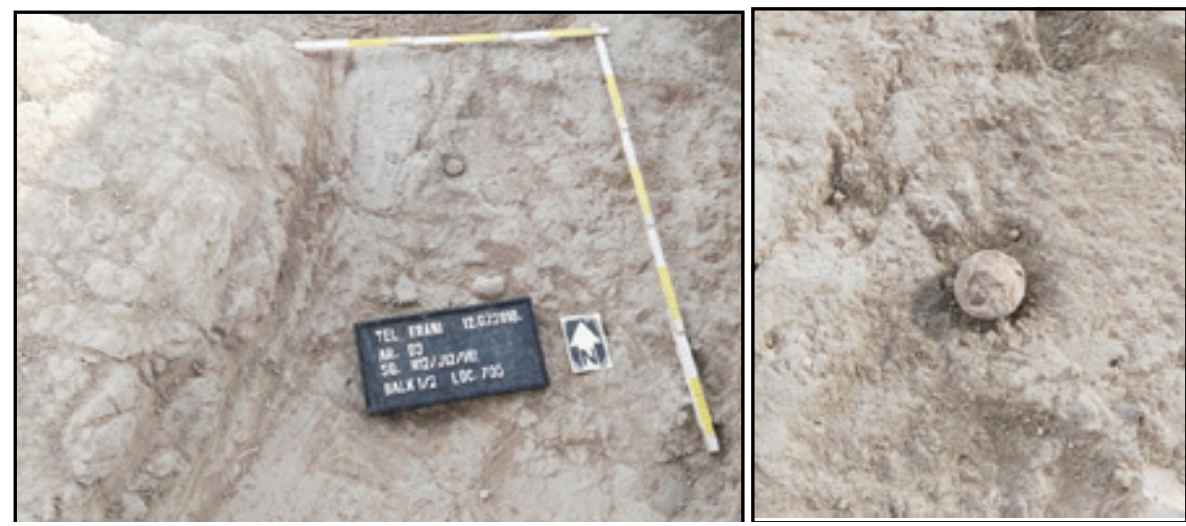

Fig. 7. Token de arcilla hallado durante la excavación de las banquetas del Área $D_{3}$ (foto: M. B. Daizo)

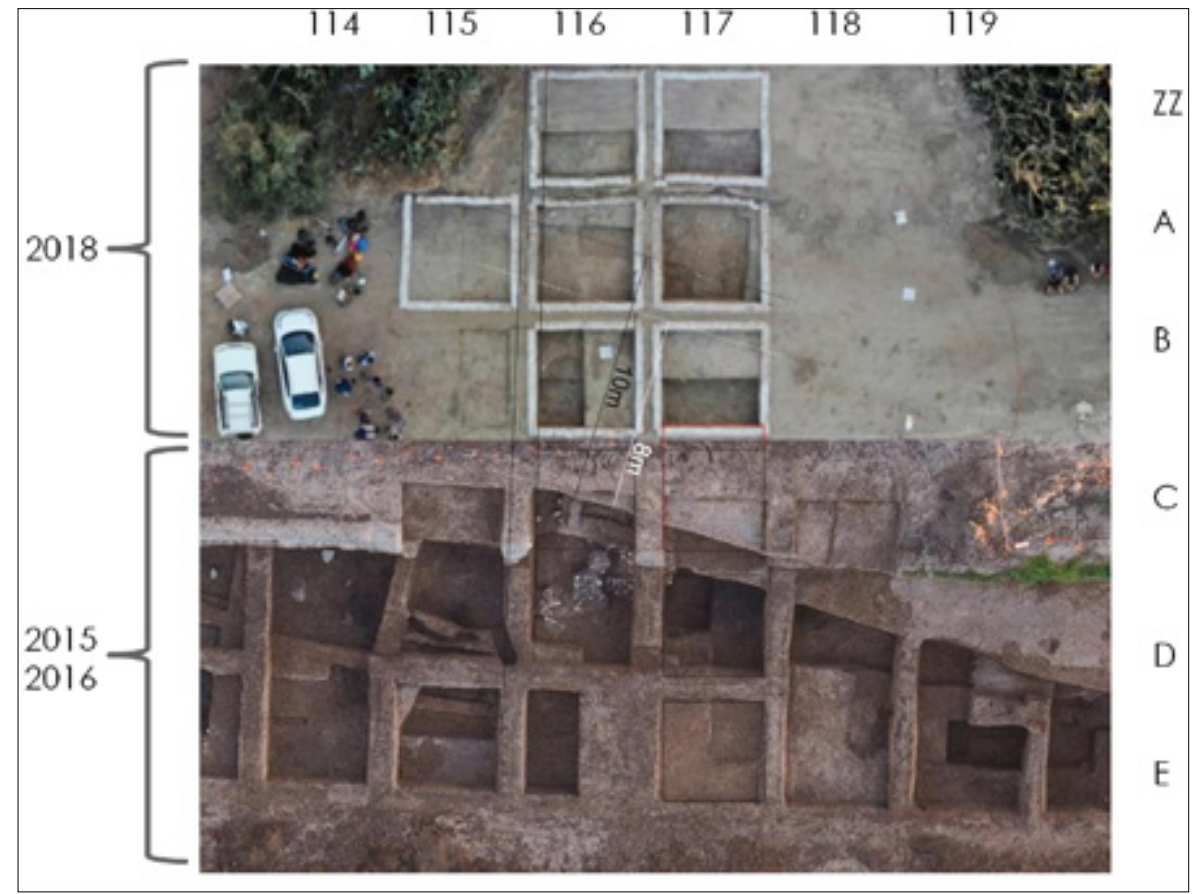

Fig. 8. Área P-Q, ubicación de las cuadrículas según las dos últimas campañas de excavación (cortesía de la Universidad Jaguelónica de Cracovia y del IAA)

Entre los hallazgos correspondientes a la fase 6 figuran, por un lado, un brazalete de cobre con tres botones, y por el otro, objetos de procedencia egipcia como madreperla (Chambardia sp.), cerámica y un fragmento de un vaso de alabastro. Se tomaron muestras de las paredes de adobe del edificio $\mathrm{H}-4$ donde fueron halladas diatomeas que muestran que los ladrillos habrían sido elaborados con lodo de orillas y humedales, ubicados en algún lugar cercano al sitio.

Los restos de la fase 7 se encontraron principalmente en la parte occidental del área excavada, consistiendo en una pared y un pozo. La pared intersecta a una banqueta entre dos cuadrículas (H11-H12), de la que se conservó sólo un curso de ladrillos, y dentro del pozo se hallaron, entre otros objetos, un pequeño sello cilíndrico con un patrón de red similar al encontrado en Tell Abu al Kharaz 
(Fischer, 2002) y un recipiente cilíndrico pulido importado, probablemente de Egipto, con una decoración interior de red (net pattern).

Con respecto a la fase 8 , perturbada por diferentes pozos, sólo se pudieron excavar las capas superiores del edificio previo, asociado posiblemente a grupos nilóticos, y con un plano similar al edificio H-4. El interior estaba ubicado en la cuadrícula J11, pero no está claro dónde se podría encontrar el borde occidental del edificio. Probablemente todavía está cubierto por las capas más recientes. Lo que sí parece evidente es que en esta fase gran parte de la cerámica fue tanto confeccionada localmente como importada de Egipto.

En resumen, las excavaciones realizadas en el Área D3H durante la última campaña arqueológica, confirman una extensa ocupación egipcia en Tel Erani. Las tres fases con evidencias de presencia nilótica muestran que no se trató de un evento único, sino prolongado en el tiempo, que podría implicar formas de relación más estables, quizás algún tipo de intercambio entre el valle del Nilo y el sur del Levante.

\section{La campaña de 2018: Área P-Q}

Como mencionamos con anterioridad, a fines de la temporada anterior se tenía la idea de que las murallas se correspondían con el Horizonte Erani C, pero como sólo se había excavado en la parte externa, todavía faltaba resolver la datación trabajando desde el interior de las mismas. Por lo tanto, el objetivo central en el Área P-Q era tratar de determinar mejor su ubicación cronológica, identificar superficies o pisos que se vinculasen a las fortificaciones desde el interior del tel. En caso de encontrar material in situ, podríamos resolver la cuestión sobre la datación de estas estructuras.

En consecuencia, se amplió la superficie de excavaciones hacia el Norte, completando un total de $200 \mathrm{~m} 2$ divididos en ocho cuadrículas. En esta área la dirección estuvo a cargo de Ianir Milevski, Marcin Czarnowicz y Eli Cohen-Sasson, desempeñándose como supervisores de cuadrícula Jacek Karmowski de la Universidad Jaguelónica de Cracovia, Sam Atkins de la Universidad Ben-Gurión del Néguev y Pablo Jaruf de la Universidad de Buenos Aires, entre otros. Desde el punto de vista metodológico, es preciso tener presente que las cuadrículas de la temporada previa habían sido tapadas por los trabajos de ensanchamiento de la ruta, por lo que se debió excavar siguiendo la red de cuadrículas anterior (Fig. 8).

Como resultado de las excavaciones se encontraron varios estratos. Los más cercanos a la superficie indican una ocupación no muy clara del BA II y III, mientras que los inferiores -muy posiblemente dos- una ocupación del BA IB1. De estos últimos, el superior cuenta con varias construcciones de ladrillos, incluyendo paredes e instalaciones circulares, además de una superficie conteniendo cerámica típica del Horizonte Erani C e instrumentos de piedra in situ (Fig. 9). La misma cubre la parte superior de una franja de $2 \mathrm{~m}$ de lo que sería la muralla superior, numerada del lado interior como W2260. Debajo de este estrato se encontró, en las cuadrículas de la parte Sudeste, la muralla mencionada, así como también una 
serie de sedimentos producto quizás de la destrucción del estrato inferior, con una superficie o piso que está adherida a W2260 desde el interior. Este piso contiene por lo menos una tinaja o ánfora y otros tiestos del Horizonte Erani C (Fig. 10).

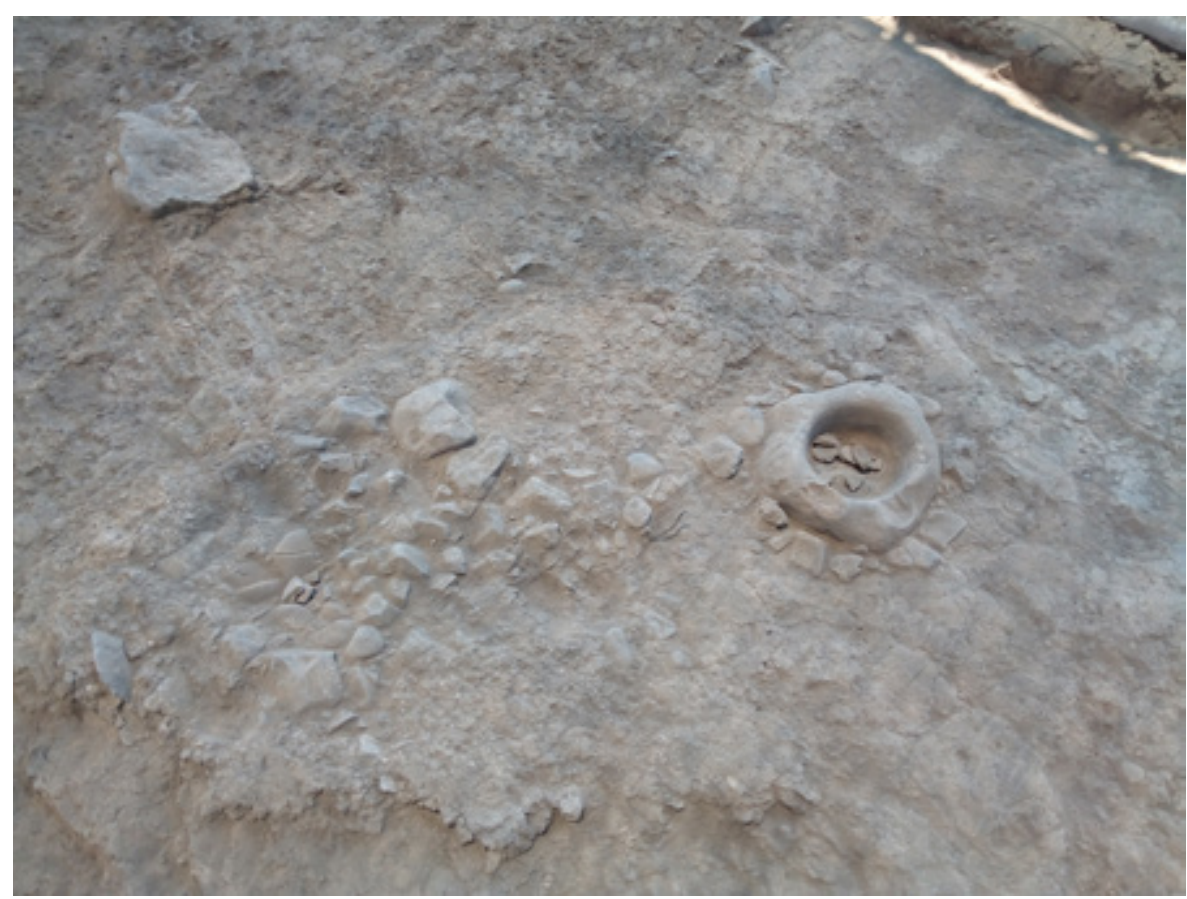

Fig. 9. Mortero y artefactos líticos in situ, cuadrícula A117, Área P-Q (foto: P. Jaruf)

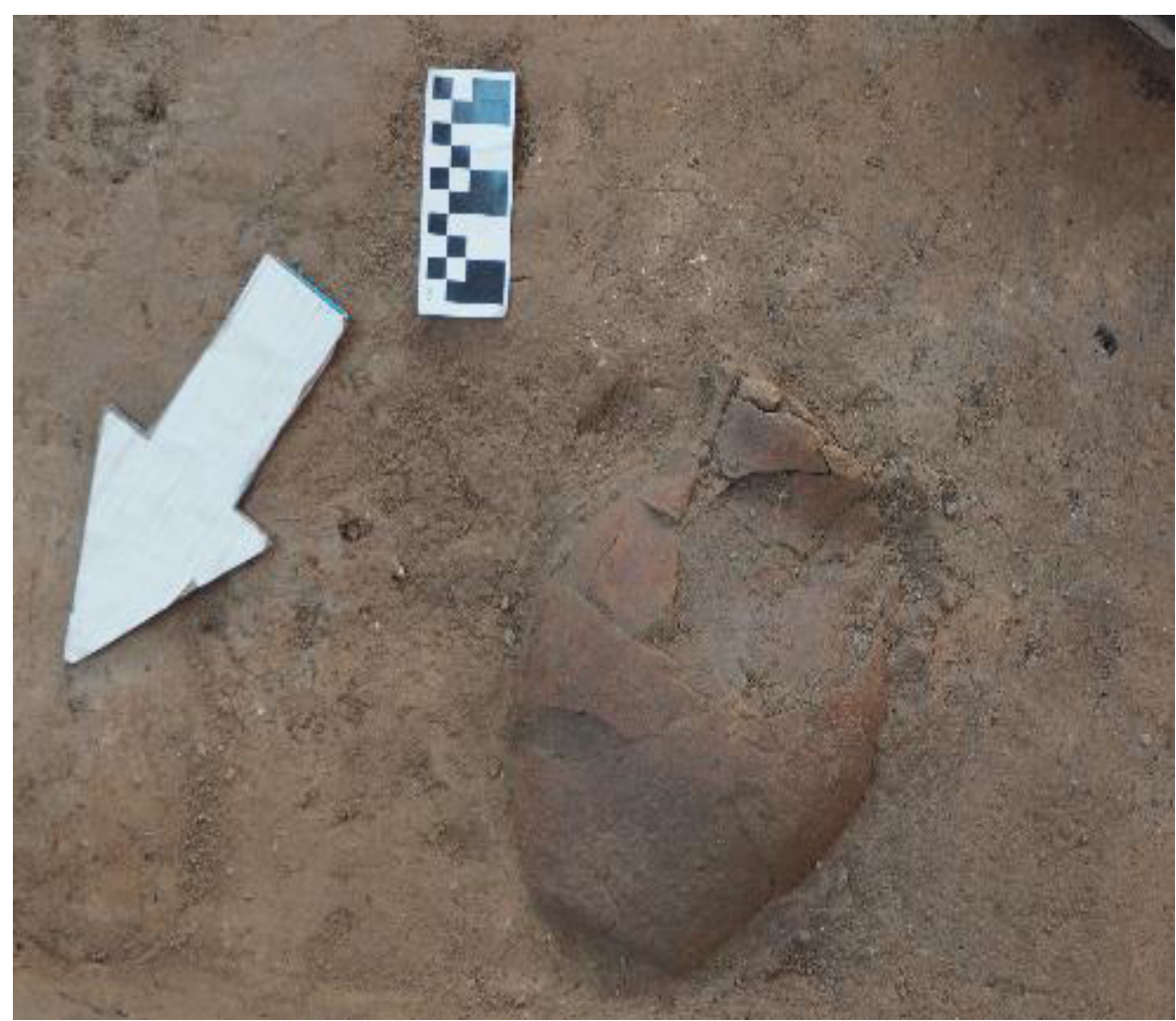

Fig. 10. Vasija estilo Erani $C$ in situ, cuadrícula A117, Área P-Q (cortesía del IAA y Universidad Jaguelónica de Cracovia) 

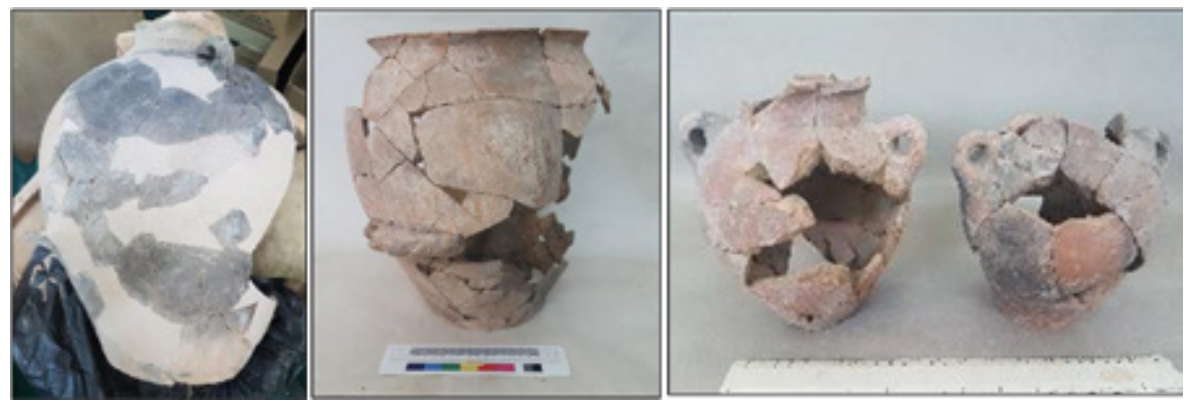

Fig. 11. Vasijas estilo Erani C, Área P-Q (cortesía del IAA)

A pesar de que aún no hemos podido comprender cabalmente la forma de las fortificaciones en esta parte de la excavación, gracias a la ilustración de una sección (véase la Fig. 5) podemos identificar cómo las superficies con material Erani $\mathrm{C}$ se relacionan con las murallas, lo cual refuerza la datación de estas estructuras como parte del BA IB1. Tomando en cuenta las dataciones por C14 mencionadas con anterioridad, podemos suponer entonces que las murallas fueron construidas alrededor del 3200-3150 a.C., lo cual implica que el BA IB1 se extendería un poco más en el tiempo, como señalan fechas de otros sitios, por ejemplo Tel Yarmuth (Regev et al., 2012), retrasando por lo tanto la presencia egipcia en la región.

En términos generales, los repertorios cerámicos hallados en esta área durante la campaña 2018 se componen por una serie de tipos bien definidos (Fig. 11): decoraciones incisas en el cuello de las vasijas y asas dobles; tinajas con decoración en líneas rojas sobre blanco llamada "pijama"; ánforas (amphoriskoi) o pequeñas tinajas con engobe rojo y pequeños utensilios en forma de lágrima o pera.

Por su parte, los instrumentos de pedernal corresponden a las características láminas de tecnología "cananea" y raspadores, a los que se agregan algunos utensilios que podrían ser de inspiración egipcia. Otros hallazgos incluyen objetos ovales de arcilla llamados (tokens), figurinas zoomorfas y, por último, huesos de animales y conchillas de moluscos marinos y del Nilo. Con respecto a esto último, nótese que podemos constatar la existencia de vínculos con material proveniente de Egipto que antecederían la fase egipcia en este sitio y serían la contrapartida de la abundante presencia de cerámica del Horizonte Erani C en el delta y en Abidos (Czarnowicz, 2018).

\section{Conclusiones preliminares}

Las excavaciones realizadas durante las últimas temporadas en Tel Erani revelaron nuevas evidencias de los procesos sociales acontecidos hacia fines del IV ${ }^{\circ}$ milenio a.C. en el sur del Levante.

Durante la exploración del Área D3 se hallaron depósitos con cuantiosos elementos egipcios que reafirman la hipótesis -particularmente si consideramos 
el número de estratos en $\mathrm{D} 3 \mathrm{H}$ - de que grupos de origen nilótico habrían permanecido en Tel Erani al menos durante un considerable período de tiempo. Probablemente sus actividades habrían estado relacionadas, como indica la evidencia del edificio $\mathrm{H}-4$, con prácticas asociadas al intercambio de bienes. Esto se desprende de los estilos cerámicos, principalmente destinados al almacenamiento, y de los tokens allí encontrados. Durante su permanencia en el sitio, los grupos nilóticos habrían conservado sus distintivas prácticas culinarias, lo que se ve reflejado en la producción de numerosas hogazas de pan y conjuntos cerámicos de vajilla utilitaria característicos de Nagada, que también han sido ampliamente registrados en el sitio de 'En Besor (Gophna, 1995).

En relación con las fortificaciones, la datación de los muros de ladrillos del Área P-Q concuerda con las sugeridas un tiempo atrás para el Área N, lo que permite plantear que el sitio de Tel Erani estuvo amurallado hacia fines del BA IB1, probablemente alrededor del 3200 a.C. o quizás un poco después. De ser así, Tel Erani constituiría el primer asentamiento amurallado en el Centro-Sur de Canaán, sumándose a otros sitios con fortificaciones ubicados en el Norte, como Ein Zippori en la Galilea (Milevski y Getzov, 2014), Bet Yerah en la costa del Mar de Galilea (Getzov, 2006), Tel Shalem y Tel Abu al Kharaz al Este y Oeste del valle del Jordán (Eisenberg, 1996; Fischer, 2008). Es posible que a este escenario se sume también Ein Asawir (Y. Paz, comunicación personal), ubicado en la llanura costera central, $115 \mathrm{~km}$ al Norte de Tel Erani.

Si consideramos que las murallas y los edificios de aparente función pública, como los edificios de pilares excavados en el Área D y DII de Tel Erani, constituyen signos de urbanismo, entonces podríamos plantear la existencia de un proceso de urbanización que antecedería la presencia clara de poblaciones egipcias en el Centro-Sur de Palestina. Sobre la extensión de las murallas, podemos también especular que cubrían todo el borde de la terraza inferior del $t e l$, rodeando el asentamiento.

No conocemos los motivos que llevaron a la construcción de murallas en Tel Erani, pero si sabemos que se superponen a un estrato anterior que posee signos de incendio y parcial destrucción. Asimismo, tenemos la certeza de que las estructuras correspondientes a este estrato se extendían al sur de la zona fortificada, más allá de la terraza del tel, por lo que la ocupación dentro de las murallas parece haber sido más pequeña que la anterior. Por último, creemos que el sitio se habría reducido aún más en el BA IB2. Los pozos de desperdicio en los que se halló material egipcio, encontrados en el Área P-Q y los observados tiempo atrás en sondeos realizados al $\mathrm{O}$ del Área $\mathrm{N}$, a cargo de Vladik Lifshits (Pasternak et al., 2016) demostrarían que los habitantes de esta fase tardía desechaban sus desperdicios fuera de la zona de habitación.

En resumen, el conjunto de toda esta evidencia nos lleva a plantear que el proceso de urbanización en Palestina parece haber comenzado antes de la presencia egipcia en la región y que pudo estar relacionado con enfrentamientos violentos, como indican el incendio y la posterior construcción de murallas acompañada de una contracción del asentamiento. Esta situación implica poner en primer plano las causas internas del proceso, quizás vinculadas con la intensificación de la producción y la circulación de bienes, principalmente intrarregional, que 
se verifica en distintos sitios del BA IA (Milevski, 2011: 198-239). Lo anterior no implica la inexistencia de vínculos con Egipto durante esta época; al contrario, mientras que en los estratos del BA IB1 identificamos bienes de procedencia egipcia, en el Alto Egipto se encuentra cerámica Erani C. No obstante, sí parecen haber cambiado las características de esta relación luego de la emergencia de los primeros asentamientos amurallados en Palestina, pues los egipcios se habrían visto interesados en asentarse en la región, quizás para tener acceso y/o un control más directo sobre las rutas de intercambio. Esto puede ser evidenciado en el Área D3, donde la presencia de grupos de origen nilótico y su permanencia durante un considerable período de tiempo es ampliamente registrada durante el BA IB2/Nagada IIIB-C.

\section{Agradecimientos}

Además de las instituciones y personas mencionadas en el reporte, nos gustaría agradecer de manera especial a las siguientes personas que han ayudado en el trabajo haciendo más amena nuestra labor (por orden alfabético): Emil Aladjem, Yasser Alamor, Sam Atkins, Eliot Braun, Krzyztof Cialowicz, Vladik Lifshits, Martin Pasternak, miembros del laboratorio de fauna, lítica y otros investigadores y estudiantes de la Universidad Jaguelónica, estudiantes de la Universidad Ben-Gurión, obreros y voluntarios de la zona. 


\section{O Bibliografía}

»Anđelković, B. (1995). The Relation Between Early Bronze Age I Canaanites and Upper Egyptians. Belgrado: Univerzitet u Beogradu, Filozofski fakultet, Centar za arheološka istraživanja.

"Brandl, B. (1989). Observations on the Early Bronze Age Strata of Tel Erani, en: Mirochedji, P. de (ed.), L'Urbanisation de la Palestine à l'Age du Bronze Ancien. Bilan et perspectives des recherches actuelles II. British Archaeological Reports International Series 527. Oxford: Archaeopress, 357-388.

»Braun, E. (2009). South Levantine Early Bronze Age Chronological Correlations with Egypt in Light of the Narmer Serekhs from Tel Erani and Arad: New Interpretations, en: British Museum Studies in Ancient Egypt and Sudan 13: 25-48.

»Czarnowicz, M. (2016). Egyptian Pottery of Tel Erani Area D-3, en: Ciałowicz, K. M., Yekutieli, Y. y Czarnowicz, M. (eds.), Tel Erani I. Preliminary Report of the 20132015 Excavations. Cracovia: Wydawnictwo Alter, 75-84.

»Czarnowicz, M. (2018). Egypt's Foreign Relations in the 4th millennium BC. The Evidence from the Tell el-Farkha Cemetery, en: Ciałowicz, K., Czarnowicz, M.

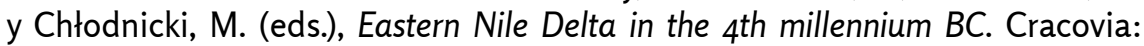
Institute of Archaeology, Jagiellonian University, 99-105.

»Czarnowicz, M., Yekutieli, Y., Ochal-Chzarnowicz, A. y Pasternak, D. (2016). The Excavation Area D-3, en: Ciałowicz, K. M., Yekutieli, Y. y Czarnowicz, M. (eds.), Tel Erani I. Preliminary Report of the 2013-2015 Excavations. Cracovia: Wydawnictwo Alter, 27-43.

»Dębowska-Ludwin, J., Rosińska-Balik, K., Czarnowicz, M. y Ochał-Czarnowicz, A. (2012). Trade or Conquest? The Nature of Egyptian-South Levantine Relations in Early Bronze I from the Perspective of Tell el-Farkha, Egypt and Tel Erani, Israel, en: Récherches Archéologiques NS 4: 113-122.

»Dothan, T. y Zuckermann, A. (2015). Philistia, en: Gitin, S. (ed.), The Ancient Pottery of Israel and Its Neighbors from the Iron Age through the Hellenistic Period. Vol. 1. Jerusalén: Israel Exploration Society - Israel Antiquities Authority - W.F. Albright Institute of Archaeological Research, 71-96.

»Dreyer, G. (2011). Tomb U-j the Burial of the Dynasty o at Abydos, en: Teeter, E. (ed.), Before the Pyramids. Chicago: University of Chicago, 127-136.

»Eisenberg, E. (1996). Tel Shalem: Soundings in a Fortified Site of the Early Bronze Age IB, en: 'Atiqot 30: 1-24.

» Fischer, P. M. (2002). Egyptian-Transjordanian Interaction during Predynastic and Protodynastic Times: The Evidence from Tell Abu al-Kharaz, Jordan Valley, en: van den Brink, E.C.M. y Levy, T. (eds.), Egypt and the Levant. Interrelations

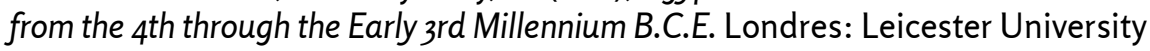
Press, 323-333.

» Fischer, P. M. (2008). Tell Abu al-Kharaz in the Jordan Valley, Volume 1: The Early Bronze Age. Contributions to the Chronology of the Eastern Mediterranean 16. Viena: Austrian Academy of Sciences.

» Getzov, N. (2006). The Tel Bet Yerah 1995 Excavations. Israel Antiquities Authority Reports 28. Jerusalén: Israel Antiquities Authority. 
» Gophna, R. (1995). Southern Canaan and the Egyptian Connection, en: Levy, T. E. (ed.), The Archaeology of Society in the Holy Land. Londres: Leicester University Press, 277-279.

»Greenberg, R. (2014). Travelling in (World) Time: Transformation, Commoditization, and the Beginnings of Urbanism in the Southern Levant, en: Wilkinson, T., Sherratt, S. y Bennet, J. (eds.), Interweaving Worlds: Systemic Interactions in Eurasia, 7 th to 1st Millennia BC. Papers from a Conference in Memory of Professor Andrew Sherratt. Oxford: Oxbow, 231-242.

» Hartung, U. (2002). Imported Jars from Cemetery U at Abydos and the Relations between Egypt and Canaan in Predynastic Times, en: van den Brink, E.C.M. y Levy, T. (eds.), Egypt and the Levant. Interrelations from the 4th through early 3 rd millennium BCE. Londres: Leicester University Press, 437-449.

» Kempinski, A. y Gilead, I. (1991). New Excavations at Tel Erani: A Preliminary Report of the 1985-1988 Seasons, en: Tel Aviv 18: 164-191.

»Kołodziejczyk, P. (2012). Tokens and Seals, en: Chłodnicki, M., Ciałowicz, K. M. y Maczynska, A. (eds.), Tell el-Farkha I. Excavations 1998-2011. Cracovia: Jagiellonian University, 267-277.

» Milevski, I. (2011). Early Bronze Age Goods Exchange in the Southern Levant. A Marxist Perspective. Londres: Equinox.

》 Milevski, I. y Getzov, N. (2014). 'En Zippori. Preliminary Report, en: Hadashot Arkheologiyot - Excavations and Surveys in Israel 126. http://www.hadashot-esi. org.il/Report_Detail_Eng.aspx?id=13675

» Milevski, I., Yegorov, D., Aladjem, E. y Pasternak, M. D. (2016). Salvage Excavation at Tel Erani, Areas P to U: Preliminary Report, en: Ciałowicz, K. M., Yekutieli, Y. y Czarnowicz, M. (eds.), Tel Erani I. Preliminary Report of the 2013-2015 Excavations. Cracovia, Wydawnictwo Alter: 45-57.

» Milevski, I., Yegorov, D., Talis, S., Nagar, Y., Smith, P. y Horwitz, L.K. (2018). The Iron Age Cemetery of Tel Erani, Socio-Cultural Diversity in the Southern Levantine Coastal Plain?, en: Stockhammer, P. y Milevski, I. (orgs.), Workshop 8: Palaeogenetics and Cultural Archaeological Perspectives on the Eastern Mediterranean Bronze and Early Iron Age, Proceedings of the 11th International Congress on the Archaeology of the Ancient Near East. Munich: LudwigMaximilians-Universität München.

》 Miroschedji, P. de (2012-2013). L'apparition des palais au Levant méridional au Bronze ancien et sa signification, en: Michel, C. (ed.), De la maison à la ville dans l'orient ancien: bâtiments publics et lieux de pouvoir. Séminaire d'Histoire et d'Archéologie des Mondes Orientaux, 2012-2013. Nanterre: Centre National de la Recherche Scientifique, 95-101.

»Pasternak, M. D., Shalev, O., Yekutieli, Y., Cohen-Sasson, E. y Atkins, S. (2016). Beyond the Wall of Tel Erani, en: Ciałowicz, K. M., Yekutieli, Y. y Czarnowicz, M. (eds.), Tel Erani I. Preliminary Report of the 2013-2015 Excavations. Cracovia: Wydawnictwo Alter, 59-63.

» Regev, J., de Miroschedji, P., Greenberg, R., Braun, E., Greenhut, Z. y Boaretto, E. (2012). Chronology of the Early Bronze Age in the Southern Levant: New Analysis for a High Chronology, en: Radiocarbon 54 (3-4): 525-566.

"Yeivin, S. (1975). El-'Areini, Tell esh Sheik Ahmed (Tel 'Erani), en: Avi-Yonah, M. (ed.), Encyclopedia of Archaeological Excavations in the Holy Land I. Jerusalén: Israel Exploration Society, 89-97. 
" Yeivin, S. (1960). Early Contacts between Canaan and Egypt, en: Israel Exploration Journal 10: 193-203.

» Yekutieli, Y. (2000). Early Bronze Age I Pottery in Southern Canaan, en: Philip, G. y Baird, D. (eds.), Ceramics and Change in the Early Bronze Age of the Southern Levant. Levantine Archaeology 2. Sheffield: Sheffield Academic Press, 129-153.

» Yekutieli, Y. (2006). The Ceramics of Tel 'Erani, Layer C, en: Glasnik, The Journal of the Serbian Archaeological Society 22: 225-242. 